\title{
Coût dans le secteur de la distribution d'électricité: économies d'échelle, économies de densité et formes institutionnelles ${ }^{1}$
}

\author{
Evrard Olivier et Lejeune Bernard ${ }^{2}$ \\ CIRIEC, Université de Liège \\ Boulevard du Rectorat, 7, B33 \\ 4000 Liège Belgium \\ E-mail : B.Lejeune@ulg.ac.be
}

Version finale

(Septembre 1998)

\begin{abstract}
Résumé
Cet article a pour objet d'évaluer empiriquement la pertinence économique (en termes d'efficience-coût) de la politique de délégation de la distribution d'électricité des communes vers des organismes ou sociétés extra-communales. Pour ce faire, les possibilités d'économies d'échelle et de densité au sein du secteur ainsi que les coûts d'exploitation relatifs des diverses formes institutionnelles existantes sont évalués sur base de l'estimation d'une fonction de coût hédonique à court terme.

Codes JEL: D20, L94.
\end{abstract}

\footnotetext{
${ }^{1}$ Cette étude a été réalisée avec le soutien financier du Fond de la Recherche Fondamentale Collective (convention FRFC n 2.4505.91 et 2.4621.92) et des Services de Programmation de la Politique Scientifique (Programme de recherche en sciences sociales, sous-programme "institutions publiques: légitimité, effectivité et efficacité") du Gouvernement belge. Elle s'insère également dans la recherche sur "les entreprises locales mixtes et le rôle des pouvoirs publics locaux" réalisée à la demande de Monsieur le Ministre G. Mathot (Région wallonne).

${ }^{2}$ Les auteurs tiennent à remercier toutes les personnes qui, de près ou de loin, ont collaboré à cette étude. En particulier, nous voudrions remercier Messieurs O. Donni et B. Thiry, ainsi que deux rapporteurs anonymes, pour leurs commentaires constructifs sur une première version de ce papier.
} 


\section{Introduction}

En Belgique, comme d'ailleurs dans nombreux autres pays européens, les activités de distribution d'électricité, d'eau, du gaz et la télédistribution sont des monopoles communaux. Le caractère de monopole communal de ces activités de distribution implique, au moins en théorie, que le pouvoir de décision ultime en ces matières est dans les mains des communes. Il ne signifie cependant nullement que les communes doivent assurer elles-mêmes ces services. Dans les faits, sous l'impulsion de leur pouvoir de tutelle, celles-ci confient généralement la gestion et l'exploitation des services de distribution à diverses formes d'organismes extérieurs qui agissent en leur nom: entreprises publiques, entreprises privées, intercommunales pures et mixtes, régies communales.

D'un point de vue économique, la justification majeure d'une telle politique de délégation - qui a pour effets d'accroître le degré de concentration (horizontale) du secteur et d'affecter sa structure institutionnelle - repose sur des considérations d'efficience-coût. L'objet de la présente étude est de vérifier le contenu empirique de cette justification dans le secteur de la distribution d'électricité.

Plus précisément, notre objectif est d'apporter des éléments de réponses à deux questions fondamentales. Premièrement, l'accroissement de la taille moyenne des distributeurs (résultat du phénomène de concentration) est-elle de nature à diminuer les coûts moyens du secteur? En d'autres termes, y a-t-il place pour des économies d'échelle - concept à différencier de celui d'économies de densité — dans ce secteur? Deuxièmement, l'efficience-coût des diverses formes institutionnelles présentes dans le secteur est-elle semblable? En d'autres termes, certaines formes institutionnelles présentent-elles systématiquement des coûts plus limités que les autres?

Accessoirement, nous nous intéressons à une troisième question, à savoir la possibilité d'économies de gamme au sein des organismes distributeurs, qui cumulent fréquemment deux activités de distribution. A ce niveau, il s'agit de vérifier si la poursuite simultanée de diverses activités (distribution d'électricité et télédistribution, distribution d'électricité et distribution du gaz) a un impact favorable économies de gamme - sur leurs coûts.

Les réponses à ces questions contiennent d'importantes implications en termes de politique économique. Ainsi, la question des économies d'échelle apporte des éléments d'informations sur la taille optimale que devraient adopter les organismes du secteur. De même, la question de l'efficience-coût relative des diverses formes institutionnelles apporte des éléments d'informations quant au choix optimal, en particulier dans le cadre du sempiternel débat secteur privé/public, de la structure institutionnelle du secteur.

Le traitement de ces questions nécessite que l'on modélise de la façon la plus crédible possible les coûts du secteur. Nous avons choisi de nous appuyer sur une fonction de coût hédonique à court terme. Le recours à une fonction de coût hédonique (c'est-à-dire basée sur une représentation hédonique de l'output) est justifié par deux types de considérations. D'une part, ce type de fonction constitue un moyen commode pour prendre en compte la nature multidimensionnelle de l'output du secteur. D'autre part, et ceci est intimement lié au point précédent, il permet de distinguer clairement les concepts d'économies d'échelle et d'économies de densité, 
distinction absolument cruciale dans l'étude des secteurs basés sur l'exploitation d'un réseau. De son côté, le recours à une fonction de coût à court terme (coût variable, représentant l'écrasante majorité des coûts d'exploitation courants) peut être justifié soit par l'existence de rigidités d'ajustement des facteurs à court terme, soit, en l'absence de telle rigidité, par la nature des données disponibles (données sur les quantités de facteurs utilisées plutôt que sur leur prix).

La mise en oeuvre du canevas décrit ci-dessus s'appuie la spécification d'une fonction de coût flexible de type translog dont les paramètres sont estimés par la méthode du maximum de vraisemblance sur des données de type panel recueillies par nos soins auprès des organismes distributeurs wallons et bruxellois. Cette banque de données contient des informations financières (coûts désagrégés) et physiques (caractéristiques du réseau, volumes d'énergie fournis, etc...). Pour des raisons liées tant aux résistances de certains organismes distributeurs à transmettre leurs informations statistiques qu'au nombre substantiel de valeurs manquantes contenues dans notre base de donnée, la présente étude porte sur un nombre très réduit d'observations.

Notre papier s'articule de la façon suivante. La Section 2 expose les fondements théoriques de notre modélisation - fonction de coût hédonique à court terme des coûts du secteur. La Section 3 est consacrée à un bref aperçu descriptif du secteur ainsi qu'à une présentation générale de nos données. La Section 4 présente la méthodologie et les résultats de notre analyse économétrique. Enfin, la conclusion résume brièvement nos principaux résultats.

\section{Fondements théoriques}

Dans le domaine de la production, la théorie de la dualité énonce les conditions sous lesquelles un processus technologique - ici, le processus de production "distribution d'électricité" — peut être indifféremment représenté par une fonction de transformation ou par une fonction de coût. Plus précisément, si on suppose qu'un processus technologique quelconque peut être représenté par la fonction de transformation

$$
F\left(y_{1}, \ldots, y_{j}, \ldots, y_{l}, x_{1}, \ldots, x_{i}, \ldots, x_{k}\right)=0
$$

où les $y_{j}$ désignent les $l$ outputs et les $x_{i}$ les $k$ inputs du processus de production, la théorie de la dualité démontre que, dans l'hypothèse où les firmes sont "preneur de prix" (price-taker) sur le marché des inputs et minimisent leur coût de production par rapport à l'ensemble de leurs inputs, le processus technologique (1) peut être décrit de manière unique et totalement équivalente par une fonction de coût total de la forme

$$
C T=G\left(y_{1}, \ldots, y_{j}, \ldots, y_{l}, p_{1}, \ldots, p_{i}, \ldots, p_{k}\right)
$$

où les $p_{i}$ désignent les prix des $k$ inputs $x_{i}$ et $C T=\sum_{i=1}^{k} p_{i} x_{i}$. Cette fonction, duale de (1), satisfait certaines propriétés. En particulier, elle est concave et homogène de degré 1 par rapport au prix des inputs.

Ainsi, sous les hypothèses énoncées, la connaissance de la seule fonction (2) permet d'inférer l'ensemble des propriétés économiquement pertinentes du processus technologique (1). De même, la fonction (1) contient toute l'information relative aux 
propriétés de (2).

D'un point de vue empirique, le choix de l'estimation de l'une ou l'autre forme dépend essentiellement des hypothèses retenues quant au caractère exogène - une donnée pour la firme - des inputs ou des outputs de son activité ${ }^{3}$. Si, comme cela est le cas dans le secteur de la distribution d'électricité, c'est l'output de l'activité qui présente un caractère exogène pour les firmes - les sociétés distributrices sont tenues de satisfaire les besoins énergétiques des ménages ou industries établis dans la zone qu'elles couvrent -, la théorie économétrique indique que seule la forme (2) peut être estimée valablement ${ }^{4}$.

\subsection{Fonction de coût hédonique, économies d'échelle et économies de densité}

La mise en oeuvre de l'approche décrite ci-dessus nécessite bien entendu une identification des inputs et des outputs du processus technologique "distribution d'électricité". En schématisant, on peut distinguer trois grands types d'inputs: le capital (représenté par le réseau), le travail et les inputs intermédiaires (essentiellement les achats d'énergie). La caractérisation de l'output est quant à elle nettement moins évidente. En effet, la distribution d'électricité est une activité où sont approvisionnés en énergie, via un réseau, un grand nombre de consommateurs. On peut dès lors considérer chaque combinaison de $\mathrm{kWh}$ fourni et de client approvisionné comme un output distinct. Sous forme vectorielle, l'output d'une firme peut donc s'écrire

$$
\mathbf{Y}=\left(y_{1}, \ldots, y_{j}, \ldots, y_{N}\right)
$$

où $y_{j}$ désigne la quantité d'énergie livrée au consommateur $j$, la dimension du vecteur Y étant égale au nombre de consommateurs.

Il est évidemment impossible d'estimer économétriquement une relation incluant un nombre de paramètres aussi important que le nombre de consommateurs desservis. Il est donc nécessaire de recourir à l'une ou l'autre forme d'agrégation du vecteur d'outputs $\mathbf{Y}$.

Dans la littérature économique traitant du secteur de la distribution d'électricité, on peut trouver deux grandes formes d'agrégation.

La première consiste à utiliser purement et simplement comme mesure agrégée de l'output, et donc comme mesure de la taille de la firme, soit le nombre total de $\mathrm{kWh}$ fournis ${ }^{5}$, soit le nombre total de consommateurs desservis ${ }^{6}$. Il s'agit ainsi simplement d'une somme (pondérée) des composantes de Y. Cette procédure d'agrégation pose d'insurmontables problèmes de mesure et d'interprétation des concepts-clés décrivant la structure d'un secteur, comme par exemple la mesure des économies d'échelle ${ }^{7}$.

\footnotetext{
${ }^{3}$ Dans la mesure où il n'existe guère de méthodes satisfaisantes pour estimer des fonctions de production multi-output, on notera que ce choix ne s'offre vraiment que dans le cas où il n'y a qu'un output.

${ }^{4}$ Pour une discussion de ce problème, voir Zellner et alii (1966).

${ }^{5}$ Voir Heuttner et alii (1978) ou Wyatt et alii (1989).

${ }^{6}$ Voir Weiss (1975) ou encore Neuberg (1977).

${ }^{7}$ Voir Panzar (1989).
} 
La seconde se rattache à ce qu'il est convenu d'appeler une représentation hédonique de l'output. Cette approche - une mesure hédonique de l'output — fut introduite dans la littérature traitant de la production par Spady et Friedlander (1978). Il s'agit d'une approche médiane entre une mesure scalaire de l'output évoquée ci-dessus et la représentation vectorielle multi-output (3). L'idée de base en est d'approximer "l'output effectif" d'une firme multi-produit par une fonction scalaire d'un nombre limité de "caractéristiques" $c_{i}$ qui résument de manière pertinente son activité. Formellement, le vecteur $\mathbf{Y}$ est donc approximé par une fonction

$$
y=C\left(c_{1}, \ldots, c_{i}, \ldots, c_{l}\right)
$$

Cette approche a largement été utilisée dans l'étude de secteurs aussi différents, quoique tous basés sur l'exploitation d'un réseau, que les chemins de fer ${ }^{8}$, les transports aériens ${ }^{9}$, ou encore la distribution d'électricité ${ }^{10}$.

Le choix des "caractéristiques" $c_{i}$ dépend bien entendu du secteur d'activité traité. Il dépend également des propriétés du processus technologique que l'on entend plus particulièrement étudier. Dans le cas qui nous occupe, une spécification intéressante de (4) est

$$
y=C(Y, N)
$$

où $Y=\sum_{i=1}^{N} y_{i}$ désigne la quantité totale d'énergie délivrée aux clients et $N$ le nombre de consommateurs desservis par le réseau. Il s'agit de la spécification retenue par Salvanes et alii (1990).

Sur base de cette spécification et en distinguant les trois inputs évoqués ci-dessus, la fonction de coût total (2) s'écrit

$$
C T=G\left(C(Y, N), p_{K}, p_{L}, p_{I}\right)=H\left(Y, N, p_{K}, p_{L}, p_{I}\right)
$$

où $p_{K}, p_{L}, p_{I}$ indiquent respectivement le prix du capital, le prix du travail et le prix des inputs intermédiaires, et comme précédemment, $C T=\sum_{i=1}^{3} p_{i} x_{i}$.

L'intérêt majeur de cette spécification est qu'elle introduit une distinction entre la taille de la firme - qui devient une notion bidimensionnelle — et la quantité d'output physique (kWh d'énergie) qu'elle délivre, distinction totalement éludée par les agrégations scalaires évoquées ci-dessus. Cette distinction permet en effet de définir deux concepts importants dans la caractérisation du secteur : les rendements d'échelle et les rendements de densité. Les rendements d'échelle (RE) mesurent l'accroissement équiproportionnel des outputs $(Y$ et $N)$ rendu possible par une augmentation équiproportionnelle de tous les inputs. Pour leur part, les rendements de densité (RD) mesurent l'accroissement relatif de l'output physique $Y$ ( $N$ étant maintenu constant) rendu possible par une augmentation équiproportionnelle de tous les inputs.

Les définitions ci-dessus sont basées sur les propriétés de la fonction de transformation (1). Dans le cadre de la théorie de la dualité, ces mesures peuvent, de manière équivalente, être dérivées de la fonction de coût total (6). Ainsi, on peut

\footnotetext{
${ }^{8}$ Voir Caves et alii (1981).

${ }^{9}$ Voir Caves et alii (1984).

${ }^{10}$ Voir Salvanes et alii (1990) et Wyatt et alii (1989).
} 
démontrer ${ }^{11}$ que

$$
\mathrm{RE}=\left(\frac{\partial \ln C T}{\partial \ln Y}+\frac{\partial \ln C T}{\partial \ln N}\right)^{-1} \quad \mathrm{RD}=\left(\frac{\partial \ln C T}{\partial \ln Y}\right)^{-1}
$$

La mesure des économies d'échelle constitue un outil essentiel pour l'évaluation d'une politique d'intégration horizontale du secteur. Elle indique les gains théoriques potentiels - en termes de coût moyen — que l'on peut attendre d'un accroissement de la taille — output physique et réseau — des firmes, c'est-à -dire de leur éventuelle fusion. Il convient d'insister sur le fait qu'il s'agit d'un gain théorique. Dans la pratique, la fusion de deux firmes ne se résume bien entendu pas à une simple addition de leur output. De son côté, la mesure des économies de densité révèle l'impact probable sur les coûts moyens de l'accroissement des consommations d'énergie des usagers. On rappellera à ce propos que, en Belgique, celles-ci se sont accrues de plus de $50 \%$ entre 1982 et 1992 . Cet indicateur est donc loin d'être sans intérêt.

\subsection{Fonction de coût total et fonction de coût variable}

Dans la discussion qui précède, nous avons supposé que les firmes minimisaient leur coût de production par rapport à l'ensemble de leurs inputs. Une telle situation est généralement qualifiée de situation de long terme. D'un point de vue empirique, pour être vérifiée, elle requiert que, dans l'intervalle d'observation d'une année qui est le nôtre, les firmes aient le temps, suite à des modifications du niveau d'output ou de prix des inputs, d'ajuster de façon optimale la quantité de chacun de leurs inputs. Si cette hypothèse est soutenable en ce qui concerne le travail et les inputs intermédiaires, elle est par contre plus discutable en ce qui concerne le capital, essentiellement constitué du réseau, dont toute modification substantielle implique des investissements lourds et coûteux.

Une manière commode de tenir compte de ces rigidités d'ajustements est de recourir à une fonction de coût à court terme (ou coût variable) où le capital est traité comme un facteur quasi-fixe ${ }^{12}$. Dans ce contexte, les firmes sont censées minimiser leur coût de production par rapport aux seuls facteurs variables $(L$ et $I$ ), et cela conditionnellement au niveau du facteur fixe. Sous cette hypothèse, la fonction de coût variable duale de (1) s'écrit

$$
C V=H_{c v}\left(Y, N, p_{L}, p_{I}, K\right)
$$

où $p_{i}(i=L, I)$ et $K$ indiquent respectivement les prix des facteurs variables et la quantité d'input quasi-fixe et, $C V=\sum_{i=1}^{2} p_{i} x_{i}$.

Tout comme la fonction de coût total (6), la fonction de coût variable (8) contient toute l'information requise pour évaluer les propriétés du processus de production sous-jacent. En particulier, les rendements d'échelle et de densité sont égaux à ${ }^{13}$

\footnotetext{
${ }^{11}$ Voir Caves et alii $(1981,1984)$.

${ }^{12}$ Voir Caves et alii (1981) ou Nelson (1985).

${ }^{13}$ Voir Caves et alii (1981).
} 


$$
\begin{gathered}
\mathrm{RE}=\left(1-\frac{\partial \ln C V}{\partial \ln K}\right)\left(\frac{\partial \ln C V}{\partial \ln Y}+\frac{\partial \ln C V}{\partial \ln N}\right)^{-1} \\
\mathrm{RD}=\left(1-\frac{\partial \ln C V}{\partial \ln K}\right)\left(\frac{\partial \ln C V}{\partial \ln Y}\right)^{-1}
\end{gathered}
$$

Les élasticités (9) et (10) sont bien entendu des élasticités à court terme. Cependant, comme indiqué par Panzar (1989), on peut montrer qu'elles correspondent aux élasticités à long terme (7) si on admet soit que le processus de production sous-jacent est homothétique, soit que la quantité du facteur quasi-fixe $K$ est optimalement ajustée à court terme, c'est-à-dire si elle satisfait la minimisation du coût total.

Dans cette dernière éventualité, l'utilisation d'une fonction de coût variable du type de (8) pour la mesure de $\mathrm{RE}$ et $\mathrm{RD}$ ne répond plus à un impératif de prise en compte de rigidités d'ajustements à court terme. Elle se justifie simplement dans l'hypothèse où des données sur les quantités de capital utilisées sont plus facilement obtenues que des données sur son prix, ce qui est notre cas.

\section{Aperçu descriptif du secteur et données disponibles}

Au préalable, une remarque: dans cet article, la fonction de distribution d'électricité est définie au sens strict, à savoir la distribution par des organismes distributeurs à des clients ultimes uniquement. Nous ne tenons donc compte ni de l'autoconsommation des autoproducteurs, ni des fournitures directes des producteurs aux industries.

\subsection{Aperçu descriptif du secteur}

Comme nous l'avons indiqué dans l'introduction, différents types d'organismes coexistent actuellement sur le territoire belge. Leurs principales caractéristiques juridiques sont les suivantes:

- Les régies communales sont des organismes gérés de façon industrielle et commerciale en dehors des services généraux des communes. Ils ne possèdent cependant pas de personnalité juridique distincte.

- Les intercommunales sont des institutions de droit public soumises à la tutelle réglementaire des autorités régionales. Pour satisfaire des besoins communaux spécifiques, la loi permet aux communes de s'associer entre elles ou avec d'autres personnes de droit public (intercommunales pures) ou de droit privé (intercommunales mixtes).

- Les concessions sont des contrats administratifs dont la caractéristique spécifique est qu'ils sont en partie à caractère contractuel et en partie à caractère réglementaire. L'autorité concédante peut donc en changer les conditions ou y 
mettre fin de manière unilatérale.

En Belgique, la forme d'organisation en intercommunale mixte domine largement le secteur. On y dénombre actuellement 20 intercommunales mixtes (9 en Région wallonne, 2 dans la Région de Bruxelles-Capitale et 9 en Région flamande) desservant 458 communes, soit plus de $80 \%$ de la population. Pour leur part, les 9 intercommunales pures desservent $18 \%$ de la population belge tandis que les 9 régies communales n'en desservent plus que $2 \%$. La forme du service communal n'a jamais prévalu. Quant au système de la concession, s'il dominait largement le secteur avant la première guerre mondiale, il est totalement tombé en désuétude au cours des années 50 et 60 , et ce précisément au profit des intercommunales mixtes. Il n'existe d'ailleurs plus aujourd'hui qu'un seul cas de concession.

La quasi totalité des intercommunales sont des sociétés coopératives, propriétaire de leurs infrastructures et dont les associées sont, dans le cas des intercommunales pures, les communes desservies par l'intercommunale plus éventuellement d'autres partenaires publics, et dans le cas des intercommunales mixtes, outre également éventuellement d'autres acteurs publics, les communes desservies et un partenaire privé. Dans ce dernier cas, le partenaire privé assure généralement l'exploitation du réseau sur base d'un contrat garantissant au secteur public la majorité du pouvoir de décision: les communes disposent toujours de la majorité des $2 / 3$ et de la présidence dans tous les organes de gestion et de contrôle de l'intercommunale. Le partenaire privé dispose quant à lui d'un droit de veto limité destiné à lui assurer la défense de ses intérêts en tant qu'associé minoritaire.

Ainsi, ce qui distingue essentiellement d'une part les intercommunales pures et les régies - malgré leur absence de personnalité juridique propre, elles peuvent être vues comme des "intercommunales pures" composées d'une seule commune - et, d'autre part, les intercommunales mixtes, c'est la présence au sein des secondes d'un partenaire privé. Le rôle de ce partenaire privé est ambigu. Il porte en effet une double casquette: il est à la fois associé minoritaire et opérateur industriel. En tant qu'associé minoritaire, il participe tant aux décisions de gestion qu'à la récolte des dividendes engendrés par les bénéfices de l'activité de distribution ${ }^{14}$. On notera à ce propos que les bénéfices des intercommunales ne sont pas soumis à l'impôt des sociétés. Par ailleurs, en tant qu'opérateur industriel, il est un sous-traitant qui opère pour le compte de l'intercommunale. Il est clair que les objectifs des acteurs publics et privés des intercommunales sont différents. Pour les premiers, la poursuite de l'intérêt général - et donc par exemple la prise en compte des externalités liées à leur activité - est censé prévaloir tandis que pour les seconds, on peut s'attendre à ce qu'ils privilégient leur intérêt propre.

Depuis la seconde guerre mondiale, on a assisté à un spectaculaire mouvement de concentration du secteur, résultant en bonne partie de la création, puis d'un mouvement de fusion, des intercommunales mixtes. Ainsi, il ne reste plus aujourd'hui que 39 entreprises de distribution alors qu'elles étaient au nombre de 235 en 1949 (141 en 1962, 66 en 1979, et 44 en 1989). A ce propos, on notera avec intérêt que la taille moyenne des intercommunales mixtes est nettement plus importante que celle de leurs homologues publiques. On l'a dit, les 20 intercommunales mixtes - soit

\footnotetext{
${ }^{14}$ Contrairement à d'autres activités de distribution, comme par exemple la distribution d'eau, la distribution d'électricité est une activité bénéficiaire.
} 
plus ou moins $50 \%$ des organismes distributeurs du pays - desservent plus de $80 \%$ de la population.

La situation en Région wallonne et bruxelloise - le champ de notre étude - est semblable à celle qui prévaut au niveau national. Ainsi, on y dénombre actuellement 11 intercommunales mixtes (9 en Wallonie et 2 à Bruxelles), 3 intercommunales pures et 3 régies communales. Le Tableau 1 montre l'importance relative de ces différents organismes sur le territoire wallon et bruxellois en 1992.

Tableau 1: Importance relative des distributeurs en Région wallonne et bruxelloise

\begin{tabular}{lccc}
\hline \hline Type d'organisme & $\begin{array}{c}\text { Nombre } \\
\text { d'organismes }\end{array}$ & $\begin{array}{c}\text { Nombre de communes } \\
\text { desservies }\end{array}$ & $\begin{array}{c}\text { Pourcentage de la } \\
\text { population desservie }\end{array}$ \\
\hline Intercommunale mixte & 11 & 223 & $81.90 \%$ \\
Intercommunale pure & 3 & 64 & $17.41 \%$ \\
Régie communale & 3 & 3 & $0.69 \%$ \\
\hline Total & 17 & 290 & $100 \%$ \\
\hline \hline
\end{tabular}

A la lecture de ce tableau, on s'étonnera sans doute du fait que le nombre total de communes desservies par l'ensemble des distributeurs est supérieur au nombre total des communes wallonnes et bruxelloises (281 communes). Ce phénomène traduit simplement les situations où plusieurs distributeurs cohabitent sur le territoire d'une même commune. La rationalisation actuelle du secteur devrait cependant aboutir à marginaliser de plus en plus ces situations. Le Tableau 1 illustre également la prédominance du secteur mixte sur le secteur public pur: les intercommunales mixtes représentent $65 \%$ du nombre total d'organismes, desservent plus de $76 \%$ des communes et près de $82 \%$ de la population. De leur côté, les 3 intercommunales pures desservent moins de $18 \%$ de cette population dans 64 communes tandis que les trois communes desservies en régie ne représentent que $0.69 \%$ de la population.

\subsection{Echantillon et données}

Comme nous venons de le voir, le territoire wallon et bruxellois compte 17 organismes de distribution d'électricité. Pour des raisons liées tant aux résistances de certains organismes distributeurs à transmettre leurs informations statistiques qu'au nombre substantiel de valeurs manquantes contenues dans notre base de donnée, nous n'avons cependant pu incorporer dans notre échantillon que sept sociétés, dont trois sont des intercommunales mixtes. Le Tableau 2 nous permet néanmoins de constater que notre échantillon est loin d'être négligeable puisque ces sept sociétés assuraient en 1992 l'approvisionnement d'approximativement 59\% des consommations d'énergie enregistrées en Région wallonne et bruxelloise.

Du point de vue des formes institutionnelles, notre échantillon est constitué d'une régie (Wavre), de trois intercommunales pures (A.L.E., A.I.E.G. et A.I.E.S.H), et de trois intercommunales mixtes (I.E.H., I.D.E.G.et S.I.B.E.L.G.).

L'unité d'observation de notre échantillon est un organisme distributeur au cours d'une année donnée. De manière à disposer d'un échantillon de taille tolérable, 
nous avons récolté dans chaque organisme des données relatives aux cinq ${ }^{15}$ exercices comptables 1988-1992.

Tableau 2: Importance relative de l'échantillon (1992)

\begin{tabular}{lcc}
\hline \hline Sociétés retenues & $\begin{array}{c}\text { Nombre de communes } \\
\text { desservies }\end{array}$ & $\begin{array}{c}\text { Pourcentage de la } \\
\text { consommation totale }\end{array}$ \\
\hline Régie de Wavre & 1 & $0.39 \%$ \\
A.L.E. & 55 & $14.09 \%$ \\
A.I.E.G. & 6 & $0.70 \%$ \\
A.I.E.S.H. & 4 & $0.88 \%$ \\
I.E.H. & 35 & $14.93 \%$ \\
I.D.E.G. & 39 & $8.90 \%$ \\
S.I.B.E.L.G. & 8 & $18.92 \%$ \\
\hline Total échantillon & 149 & $58.84 \%$ \\
\hline Total des 2 Régions & 281 & $100.00 \%$ \\
\hline \hline
\end{tabular}

Les définitions empiriques des variables évoquées dans la Section 2, et utilisées ci-dessous dans l'analyse économétrique, sont les suivantes ${ }^{16}$ :

- $C V$ est le coût variable de distribution. Il inclut la masse salariale globale et le montant total des achats d'énergie. Ce coût représente l'écrasante majorité des coûts d'exploitation annuels courants (c'est-à-dire hors coûts fixes: amortissements, charge des dettes,...) d'un service de distribution d'électricité.

- $Y$ est la quantité totale d'énergie (MWh haute tension et basse tension) délivrée par la société.

- $N$ est le nombre total de consommateurs desservis par la société . Il inclut tant les abonnés ordinaires que les consommateurs industriels (très peu nombreux).

- $K$ est la quantité de capital employée par le distributeur. Dans la mesure où le réseau constitue l'input capital majeur de l'activité distributrice, comme variable "proxy" de cette quantité, nous avons utilisé la longueur (Kms) totale du réseau de distribution de la société.

- $p_{L}$ est le prix de l'input travail. Ce taux de salaire est défini comme la masse salariale totale divisée par le nombre total de travailleur occupé. Etant donné que les distributeurs rentrent en compétition avec les firmes des autres secteurs dans l'acquisition de leur main-d'oeuvre, ce taux de salaire est considéré comme une donnée (price-taker) pour les sociétés.

- $p_{I}$ est le prix moyen de l'énergie appelée par le distributeur. Il est défini comme le montant total des achats d'énergie divisé par la quantité totale d'énergie appelée. Dans la mesure où ce prix est déterminé sur base de contrats d'achats à long terme, il est traité, au cours de chaque période, comme une donnée (price-taker) pour la firme.

\footnotetext{
${ }^{15}$ Seules des données relatives aux quatre derniers exercices comptables étaient disponibles à la Régie de Wavre. Cela explique le nombre total de nos observations: 34 .

${ }^{16}$ Notons qu'en pratique, dans la définition de $C V$, la masse salariale globale engendrée par l'activité de distribution ne peut être qu'estimée. En effet, la masse salariale reportée dans les comptes inclut en partie des "travaux pour compte de tiers" et des "productions immobilisées", postes qui doivent être déduits des coûts d'exploitation courants.
} 
Le Tableau 3 fournit un aperçu descriptif de ces variables pour 1992.

Tableau 3: Echantillon en 1992, statistiques descriptives

\begin{tabular}{lcccc}
\hline \hline Variable & Moyenne & Ecart-type & Minimum & Maximum \\
\hline$Y$ & 1297475 & 1205309 & 61373 & 2921658 \\
$N$ & 168133 & 154986 & 8082 & 368276 \\
$K$ & 4924 & 4393 & 245 & 9972 \\
$p_{L}$ & 2024393 & 596515 & 1300762 & 2741500 \\
$p_{I}$ & 2323 & 135 & 2101 & 2465 \\
\hline \hline
\end{tabular}

Comme on peut le constater, ces données sont extrêmement dispersées. Le plus petit distributeur (Régie de Wavre) desservait en 1992 à peine 8082 consommateurs alors que le plus important (I.E.H.) en desservait 368276. La corrélation observée dans l'échantillon entre les deux outputs $Y$ et $N$ est très forte, de l'ordre de 0.96 . Les corrélations observées entre chacun de ces deux outputs et le stock de capital $K$ sont nettement plus modérées : celle entre $Y$ et $K$ est de 0.71 et celle entre $N$ et $K$ est de 0.80 .

\section{Analyse économétrique}

L'estimation économétrique de la fonction de coût hédonique à court terme (8) requiert que l'on spécifie une forme fonctionnelle pour cette relation. Elle requiert également que l'on spécifie un certain nombre d'hypothèses quant à la structure stochastique du modèle. C'est l'objet des deux sections suivantes. La troisième section reporte les résultats d'estimation.

\subsection{La fonction translog}

Comme il est coutumier de le faire dans ce genre d'étude, nous avons choisi comme forme fonctionnelle pour la relation (10) la forme flexible de type translog suivante:

$$
\begin{aligned}
\ln C V= & \alpha_{0}+\alpha_{Y} \ln Y+\alpha_{N} \ln N+\alpha_{K} \ln K+1 / 2 \alpha_{Y Y}(\ln Y)^{2}+1 / 2 \alpha_{N N}(\ln N)^{2} \\
& +1 / 2 \alpha_{K K}(\ln K)^{2}+\alpha_{Y N} \ln Y \ln N+\alpha_{Y K} \ln Y \ln K+\alpha_{N K} \ln N \ln K \\
& +\alpha_{Y I} \ln Y \ln p_{I}+\alpha_{N I} \ln N \ln p_{I}+\alpha_{K I} \ln K \ln p_{I} \\
& +\alpha_{Y L} \ln Y \ln p_{L}+\alpha_{N L} \ln N \ln p_{L}+\alpha_{K L} \ln K \ln p_{L} \\
& +\alpha_{I} \ln p_{I}+\alpha_{L} \ln p_{L}+1 / 2 \alpha_{I I}\left(\ln p_{I}\right)^{2}+1 / 2 \alpha_{L L}\left(\ln p_{L}\right)^{2} \\
& +\alpha_{I L} \ln p_{I} \ln p_{L}+\alpha_{a} \text { Dact }+\alpha_{p} \text { Dpriv }
\end{aligned}
$$

où les $\alpha_{i}$ sont les paramètres de la fonction, Dact est une variable binaire indiquant si la société poursuit ou non d'autres activités que la distribution d'électricité (gaz, télédistribution), Dpriv est une variable binaire indiquant si le capital social de la société est ou non mixte (privé et public). Les autres variables ont été définies précédemment. 
L'introduction de la variable Dact dans la relation (11) constitue un moyen simple $^{17}$ de tester la présence d'économie de gamme, c'est-à-dire des économies engendrées par la poursuite simultanée de plusieurs activités par les firmes. De son coté, l'introduction de la variable Dpriv a pour objet de vérifier si la forme institutionnelle de la société (en l'occurrence son caractère d'intercommunale mixte), influence ses coûts.

Par application du lemme de Sheppard (1970), les parts de facteur $S_{I}$ et $S_{L}$ respectivement, les achats d'énergie et la masse salariale — dans le coût variable $C V$ s'écrivent $\left(S_{I}+S_{L}=1\right)$

$S_{I}=\frac{\partial \ln C V}{\partial \ln p_{I}}=\alpha_{I}+\alpha_{I I} \ln p_{I}+\alpha_{I L} \ln p_{L}+\alpha_{Y I} \ln Y+\alpha_{N I} \ln N+\alpha_{K I} \ln K$
$S_{L}=\frac{\partial \ln C V}{\partial \ln p_{L}}=\alpha_{L}+\alpha_{L L} \ln p_{L}+\alpha_{I L} \ln p_{I}+\alpha_{Y L} \ln Y+\alpha_{N L} \ln N+\alpha_{K L} \ln K(13)$

Comme mentionné dans la Section 2, une fonction de coût duale doit satisfaire certaines propriétés, en particulier elle doit être concave et homogène de degré 1 par rapport au prix des inputs ${ }^{18}$. Dans le cas de la fonction (11) et des parts de facteur associées (12)-(13), la propriété d'homogénéité est respectée si et seulement si les contraintes suivantes sur les paramètres sont satisfaites:

$$
\begin{array}{ccc}
\alpha_{I}+\alpha_{L}=1 & \alpha_{I I}+\alpha_{I L}=0 & \alpha_{L L}+\alpha_{I L}=0 \\
\alpha_{Y I}+\alpha_{Y L}=0 & \alpha_{N I}+\alpha_{N L}=0 & \alpha_{K I}+\alpha_{K L}=0
\end{array}
$$

Dans son côté, compte tenu des restrictions (14), la concavité par rapport au prix des inputs est respectée si et seulement si

$$
\alpha_{I I}-S_{I}+S_{I}^{2} \leqslant 0
$$

Pour des raisons d'efficacité d'estimation, on estimera de manière jointe la fonction de coût (11) et, pour éviter tout problème lié à la singularité de la matrice de variance-covariance des observations, une des deux parts de facteur associées. Comme montré par Barten (1969), le choix de l'équation de part de facteur non prise en compte est sans importance. Arbitrairement, nous abandonnerons $S_{L}$.

Contrairement aux conditions d'homogénéité (14), la restriction (15) est difficile à prendre en compte lors de l'estimation. Nous suivrons l'usage qui consiste à simplement la vérifier a posteriori.

L'estimation jointe de (11) et (12) sous les contraintes (14) implique la prise en compte de restrictions intra et inter équations. Les restrictions intra-équations peuvent être facilement éliminées. En effet, les contraintes sur les paramètres (14)

\footnotetext{
${ }^{17}$ Simple mais non rigoureux. Une approche rigoureuse de ce problème nécessite en effet la spécification d'une fonction de coût incorporant simultanément toutes les activités des firmes. Une telle approche se situe cependant au delà de notre propos.

${ }^{18}$ Voir les conditions générales fournies par Diewert et alii (1987).
} 
peuvent être réécrites

$$
\begin{array}{ccc}
\alpha_{L}=1-\alpha_{I} & \alpha_{I L}=-\alpha_{I I} \quad \alpha_{L L}=\alpha_{I I} \\
\alpha_{Y L}=-\alpha_{Y I} & \alpha_{N L}=-\alpha_{N I} & \alpha_{K L}=-\alpha_{K I}
\end{array}
$$

En substituant ces restrictions dans les équations (11) et (12), on obtient la forme contrainte de la fonction de coût variable et de sa part de facteur associée

$$
\begin{aligned}
\ln \left(C V / p_{L}\right)= & \alpha_{0}+\alpha_{Y} \ln Y+\alpha_{N} \ln N+\alpha_{K} \ln K+1 / 2 \alpha_{Y Y}(\ln Y)^{2} \\
& +1 / 2 \alpha_{N N}(\ln N)^{2}+1 / 2 \alpha_{K K}(\ln K)^{2}+\alpha_{Y N} \ln Y \ln N \\
& +\alpha_{Y K} \ln Y \ln K+\alpha_{N K} \ln N \ln K+\alpha_{Y I} \ln Y \ln \left(p_{I} / p_{L}\right) \\
& +\alpha_{N I} \ln N \ln \left(p_{I} / p_{L}\right)+\alpha_{K I} \ln K \ln \left(p_{I} / p_{L}\right) \\
& +\alpha_{I} \ln \left(p_{I} / p_{L}\right)+1 / 2 \alpha_{I I}\left(\ln \left(p_{I} / p_{L}\right)\right)^{2} \\
& +\alpha_{a} \text { Dact }+\alpha_{p} \text { Driv } \\
S_{I}=\alpha_{I}+ & \alpha_{I I} \ln \left(p_{I} / p_{L}\right)+\alpha_{Y I} \ln Y+\alpha_{N I} \ln N+\alpha_{K I} \ln K
\end{aligned}
$$

Sur base de la forme fonctionnelle (17), on notera finalement que les rendements d'échelle et de densité s'écrivent

$$
\mathrm{RE}=\frac{1-E_{K}}{E_{Y}+E_{N}} \quad \mathrm{RD}=\frac{1-E_{K}}{E_{Y}}
$$

où

$$
\begin{aligned}
& E_{K}=\alpha_{K}+\alpha_{K K} \ln K+\alpha_{Y K} \ln Y+\alpha_{N K} \ln N+\alpha_{K I} \ln \left(p_{I} / p_{L}\right) \\
& E_{Y}=\alpha_{Y}+\alpha_{Y Y} \ln Y+\alpha_{Y N} \ln N+\alpha_{Y K} \ln K+\alpha_{Y I} \ln \left(p_{I} / p_{L}\right) \\
& E_{N}=\alpha_{N}+\alpha_{N N} \ln N+\alpha_{Y N} \ln Y+\alpha_{N K} \ln K+\alpha_{N I} \ln \left(p_{I} / p_{L}\right)
\end{aligned}
$$

et que si le processus de production sous-jacent est homothétique, on doit avoir ${ }^{19}$

$$
\alpha_{Y I}=\alpha_{N I}=0
$$

\subsection{Hypothèses stochastiques}

Le système formé par les équations (17) et (18) peut être écrit, en empilant correctement les variables, comme un système linéaire stochastique bivarié non contraint :

$$
\left[\begin{array}{c}
\ln \left(C V / p_{L}\right)_{i t} \\
S_{I_{i t}}
\end{array}\right]=\left[\begin{array}{c}
X_{i t}^{C V} \\
X_{i t}^{S}
\end{array}\right] \alpha+\left[\begin{array}{c}
\varepsilon_{i t}^{C V} \\
\varepsilon_{i t}^{S}
\end{array}\right]
$$

sous forme plus compacte,

$$
Z_{i t}=X_{i t} \alpha+\varepsilon_{i t} \quad i=1,2, \ldots, M ; t=1,2, \ldots, T_{i}
$$

\footnotetext{
${ }^{19}$ Voir par exemple Spady et alii $(1978)$.
} 
où les indices $i$ et $t$ désignent respectivement l'entreprise $i$ et la période d'observation $t$ ( $T_{i}$ observations par entreprise), $X_{i t}^{C V}$ est un vecteur ligne $(1 \times k)$ reprenant les variables explicatives apparaissant dans $(17), X_{i t}^{S}$ est un vecteur ligne $(1 \times k)$ composé de zéros et des variables explicatives de (18) correctement placées, $\alpha$ est un vecteur $(k \times 1)$ de paramètres non contraints - les contraintes inter-équations sont prises en compte par l'empilement des explicatives - et, $\varepsilon_{i t}^{C V}$ et $\varepsilon_{i t}^{S}$ sont les termes d'erreurs propres de chaque équation.

Adoptant la spécification proposée par Magnus (1982), on supposera que $\varepsilon_{i t}$ est égal à la somme d'un terme d'erreur propre à chaque entreprise et d'un terme d'erreur général

$$
\varepsilon_{i t}=\mu_{i}+\nu_{i t}
$$

où $\mu_{i}$ et $\nu_{i j}$ sont des vecteurs aléatoires $(2 \times 1)$ mutuellement indépendants, indépendants des variables explicatives et identiquement indépendamment distribués selon une loi normale avec une moyenne nulle et une matrice de variance-covariance constante

$$
\mu_{i} \sim \mathrm{N}_{2}(0, \Gamma) \quad \nu_{i t} \sim \mathrm{N}_{2}(0, \Sigma)
$$

Cette spécification stochastique est une généralisation au cas des données en panel - prise en compte d'une hétérogénéité non observée entre les entreprises - du modèle SURE standard de Zellner (1962). L'estimation par maximum de vraisemblance de ce modèle est décrite en détail dans Magnus (1982).

\subsection{Résultats empiriques}

Le Tableau 4 reporte les résultats de l'estimation par maximum de vraisemblance du modèle (21)-(23). Comme il est usuel de le faire dans ce type d'étude, toutes les variables sont standardisées de telle sorte que $\alpha_{Y}, \alpha_{N}, \alpha_{K}$ et $\alpha_{I}$ peuvent être directement interprétés comme les valeurs au point moyen de l'échantillon de respectivement l'élasticité du coût par rapport à $Y, N$ et $K$, et la part des achats d'énergie dans le coût variable. Ainsi, au point moyen de l'échantillon, la part estimée des achats d'énergie dans le coût variable est de $87 \%$ et celle de la masse salariale de $13 \%$. De même, toujours au point moyen de l'échantillon, un accroissement de $1 \%$ de chacune des variables $Y, N$ et $K$ induit respectivement - toutes autres choses étant égales — une hausse du coût d'exploitation de $0.81 \%, 0.09 \%$ et $0.12 \%$. Ces chiffres, qui apparaîssent tout à fait raisonnables, sont proches des résultats obtenus dans une étude similaire menée sur des données norvégiennes par Salvanes et alii (1990).

On notera la significativité de l'effet individuel aléatoire bivarié $\mu_{i}$ introduit dans le modèle. Le test de Wald ${ }^{20}$ de l'hypothèse nulle que $\Gamma=0$ rejette en effet cette hypothèse au seuil de $5.1 \%\left(\chi^{2}(3)=7.7541\right.$, Prob. $\left.=0.0514\right)$. Cela confirme la présence d'une hétérogénéité non observée entre les entreprises, hétérogénéité qui est sensiblement de même ampleur que celle du terme d'erreur général (les ordres de grandeurs de $\hat{\Sigma}$ et $\hat{\Gamma}$ sont comparables).

\footnotetext{
${ }^{20}$ Pour les détails de la mise en oeuvre de ce test un peu particulier puisqu'il s'applique aux éléments d'une matrice de variance-covariance, voir Magnus (1982).
} 
Tableau 4: Résultats d'estimation

\begin{tabular}{|c|c|c|c|c|c|c|}
\hline$\overline{\text { Variable }}^{a}$ & \multicolumn{2}{|c|}{ Coefficient } & Ecart-type & \multicolumn{2}{|c|}{ t-stat. } & Prob. \\
\hline Intercept & \multicolumn{2}{|c|}{-0.0165} & 0.0269 & \multicolumn{2}{|c|}{-0.612} & 0.5408 \\
\hline $\ln Y$ & \multicolumn{2}{|c|}{0.8098} & 0.0413 & \multicolumn{2}{|c|}{19.627} & 0.0000 \\
\hline $\ln N$ & \multicolumn{2}{|c|}{0.0923} & 0.0471 & \multicolumn{2}{|c|}{1.961} & 0.0498 \\
\hline $\ln K$ & \multicolumn{2}{|c|}{0.1222} & 0.0187 & \multicolumn{2}{|l|}{6.538} & 0.0000 \\
\hline $1 / 2(\ln Y)^{2}$ & \multicolumn{2}{|c|}{0.0585} & 0.1548 & \multicolumn{2}{|l|}{0.378} & 0.7055 \\
\hline $1 / 2(\ln N)^{2}$ & \multicolumn{2}{|c|}{-0.0336} & 0.1563 & \multicolumn{2}{|c|}{-0.215} & 0.8295 \\
\hline $1 / 2(\ln K)^{2}$ & \multicolumn{2}{|c|}{0.0508} & 0.0763 & \multicolumn{2}{|c|}{0.667} & 0.5051 \\
\hline $\ln Y \ln N$ & \multicolumn{2}{|c|}{0.0329} & 0.1435 & \multicolumn{2}{|c|}{0.230} & 0.8185 \\
\hline $\ln Y \ln K$ & \multicolumn{2}{|c|}{-0.0513} & 0.0352 & \multicolumn{2}{|c|}{-1.458} & 0.1448 \\
\hline $\ln N \ln K$ & \multicolumn{2}{|c|}{-0.0032} & 0.0676 & \multicolumn{2}{|c|}{-0.047} & 0.9622 \\
\hline $\ln Y \ln \left(p_{I} / p_{L}\right)$ & \multicolumn{2}{|c|}{0.0640} & 0.0317 & \multicolumn{2}{|c|}{2.015} & 0.0439 \\
\hline $\ln N \ln \left(p_{I} / p_{L}\right)$ & \multicolumn{2}{|c|}{0.0189} & 0.0330 & \multicolumn{2}{|l|}{0.574} & 0.5658 \\
\hline $\ln K \ln \left(p_{I} / p_{L}\right)$ & \multicolumn{2}{|c|}{-0.0700} & 0.0140 & \multicolumn{2}{|c|}{-5.003} & 0.0000 \\
\hline $\ln \left(p_{I} / p_{L}\right)$ & \multicolumn{2}{|c|}{0.8703} & 0.0072 & \multicolumn{2}{|c|}{120.821} & 0.0000 \\
\hline $1 / 2\left(\ln \left(p_{I} / p_{L}\right)\right)^{2}$ & \multicolumn{2}{|c|}{0.0535} & 0.0128 & \multicolumn{2}{|c|}{4.168} & 0.0000 \\
\hline Dact & \multicolumn{2}{|c|}{0.0118} & 0.0180 & \multicolumn{2}{|c|}{0.656} & 0.5118 \\
\hline Dpriv & \multicolumn{2}{|c|}{-0.0261} & 0.0131 & \multicolumn{2}{|c|}{-1.987} & 0.0469 \\
\hline $\begin{array}{l}\text { Paramètres de } \\
\text { Variance }\end{array}$ & $\hat{\Sigma}=$ & $\begin{array}{l}0.00058 \\
-0.00046\end{array}$ & $\begin{array}{r}-0.00046 \\
0.00038\end{array}$ & $\hat{\Gamma}=$ & $\begin{array}{l}0.00035 \\
-0.00029\end{array}$ & $\begin{array}{c}-0.00029 \\
0.00059\end{array}$ \\
\hline
\end{tabular}

${ }^{a}$ Régresseurs de la fonction de coût (17). Variables normalisées.

On peut aisément vérifier que la condition de concavité (15) est satisfaite au point moyen de l'échantillon. De façon assez remarquable, cette condition est en fait non seulement satisfaite au point moyen mais aussi en tous les points de notre échantillon. Par ailleurs, les tests de Wald des hypothèses nulles de l'absence d'effets de l'input quasi-fixe $K\left(\mathrm{H}_{0}: \alpha_{K}=\alpha_{K K}=\alpha_{N K}=\alpha_{Y K}=\alpha_{K I}=0\right)$ et l'absence d'effets de la taille du réseau $N\left(\mathrm{H}_{0}: \alpha_{N}=\alpha_{N N}=\alpha_{N K}=\alpha_{Y N}=\alpha_{N I}=0\right)$ rejettent chacune de ces hypothèses à un seuil inférieur à $0.01 \%$ (on a respectivement $\chi^{2}(5)=61.93$, Prob. $=0.0000$ et $\chi^{2}(5)=95.50$, Prob. $\left.=0.0000\right)$. Ainsi, tant la représentation hédonique de l'output du secteur par $Y$ et $N$ que la prise en compte de $K$ en tant qu'input quasi-fixe semblent constituer une modélisation pertinente du secteur.

Une partie des paramètres associés à la fonction de coût translog n'apparaissent guère individuellement significatifs: les termes quadratiques et d'interactions entre $Y, N$ et $K$ (lignes 5 à 10 du Tableau 4, soit les paramètres $\alpha_{Y Y}, \alpha_{N N}, \alpha_{K K}, \alpha_{Y N}$, $\alpha_{Y K}$ et $\left.\alpha_{N K}\right)$. Etant donné la petite taille de notre échantillon et la collinéarité importante entre ces variables, ce n'est pas très étonnant. Cela ne signifie cependant nullement qu'ils sont conjointement non significatifs. En fait, le test de Wald de la nullité jointe de ces six paramètres rejette cette hypothèse à un seuil inférieur à $0.01 \%$ $\left(\chi^{2}(6)=43.82\right.$, Prob. $\left.=0.0000\right)$. Bref, les valeurs faibles des 't-stat.' de ces variables indiquent une imprécision certaine de l'estimation des paramètres de second ordre pris individuellement, mais pas que les termes quadratiques et d'interactions entre $Y, N$ et $K$, qui permettent aux élasticités d'échelle et de densité de varier selon le niveau d'activité, peuvent être négligés. 
Comme on peut le constater, chacune des deux variables binaires introduites dans le modèle présente un coefficient ayant le signe attendu, positif pour Dact et négatif pour Dpriv, mais seul le second est significativement différent de zéro.

Ainsi, nous n'avons pas trouvé d'évidences statistiques de la possibilité d'économies de gamme au sein des sociétés poursuivant simultanément plusieurs activités de distribution. Ce résultat est plutôt contre-intuitif. En effet, la possibilité d'économies de gamme semble relativement naturelle dans le cadre d'activités de distribution. Une première explication pourrait en être la faiblesse de l'information contenue dans notre échantillon à cet égard. Une autre explication nous semble potentiellement pertinente. Rappelons que nous travaillons avec une fonction de coût variable, qui par définition exclus le coût du capital (le réseau). Or il se pourrait que ce soit précisément au sein des coûts afférents au réseau que des économies de gamme puissent apparaître. Cela semble particulièrement évident pour les sociétés assurant simultanément la distribution d'électricité et la télédistribution.

Par contre, il semble bel et bien y avoir des différences de coût d'exploitation systématiques entre les organismes purement public et les organismes mixtes. Notons cependant que cette différence est relativement modeste puisqu'elle équivaut à plus ou moins $2.5 \%$ des coûts. Nous l'avons dit, au sein des intercommunales mixtes, le partenaire privé ne poursuit pas nécessairement les mêmes objectifs que ses associés publics. C'est d'ailleurs notamment pour cette raison que le législateur a statutairement limité son pouvoir (Cfr. Section 3.1). Si l'on admet que le partenaire privé a pour objectif de maximiser ses profits propres, il peut soit tenter de gonfler la facture qu'il présente à l'intercommunale mixte en tant que sous-traitant, ce qui diminuera cependant d'autant les bénéfices de l'intercommunale, et donc les dividendes qu'il en obtiendra en tant qu'actionnaire minoritaire, soit au contraire participer "loyalement" à la maximisation des profits de l'intercommunale, et donc de baser ses propres profits sur les dividendes qu'il pourra en obtenir. Dans cette deuxième hypothèse, sachant qu'il n'a d'influence ni sur la demande, ni sur le prix de vente de l'énergie - celui-ci est fixé par un organisme national et s'impose à tous les distributeurs - il n'a comme solution pour atteindre son objectif que d'essayer de peser sur les coûts d'exploitation, soit directement, soit indirectement par l'intermédiaire d'une baisse de la qualité du service offert (rappelons que les clients sont ici captifs).

La première stratégie semble difficile à mettre en oeuvre car les partenaires publics (essentiellement les communes) de l'intercommunale dispose, comme on l'a vu, d'un pouvoir de contrôle important. Par ailleurs, cette stratégie rentre en conflit direct avec leurs intérêts financiers (les communes tirent un revenu non négligeable de la distribution d'électricité). Reste la deuxième, dont on notera au passage qu'elle est encouragée par le régime fiscal favorable dont bénéficie les intercommunales (Cfr. Section 3.1). Le présent résultat semble indiquer que la pression à la baisse sur les coûts est bien réelle. Nos données ne nous permettent cependant pas de dire si c'est ou non au détriment de la qualité (fréquence des ruptures d'approvisionnement, rapidité des réparations en cas de rupture d'approvisionnement,...). Une telle baisse de qualité pourrait par exemple provenir d'un sous-investissement dans l'infrastructure. A ce niveau, les associés privés des intercommunales mixtes ont clairement intérêt à s'en tenir au strict minimum. 
Il nous reste à examiner les valeurs des rendements d'échelle et de densité impliquées par les paramètres estimés repris au Tableau 4. Le Tableau 5 résume les résultats obtenus à cet égard. Il reporte les rendements d'échelle et de densité calculés à trois points typiques de l'échantillon: au point moyen (168133 abonnés), pour la plus petite société (Wavre en 1992, 8082 abonnés) et pour la plus grande société (I.E.H. en 1992, 368276 abonnés).

Tableau 5: Rendements d'échelle et rendements de densité

\begin{tabular}{lcccc}
\hline \hline & & Valeur & Ecart-type & $\begin{array}{c}\text { Intervalle de } \\
\text { confiance à 95\% }\end{array}$ \\
\hline \multirow{2}{*}{ Rendements d'échelle } & Wavre & 1.0819 & 0.0418 & $0.9999-1.1638$ \\
& Pt. moyen & 0.9731 & 0.0173 & $0.9391-1.0071$ \\
& I.E.H. & 0.9564 & 0.0310 & $0.8955-1.0172$ \\
\multirow{2}{*}{ Rendements de densité } & Wavre & 1.2527 & 0.0988 & $1.0590-1.4464$ \\
& Pt. moyen & 1.0840 & 0.0528 & $0.9804-1.1876$ \\
& I.E.H. & 1.0475 & 0.0934 & $0.8644-1.2306$ \\
\hline \hline
\end{tabular}

La première colonne du Tableau 5 donne la valeur des rendements d'échelle et de densité calculés sur base des expressions (19). La seconde colonne fournit l'écarttype de ces valeurs. Enfin, la dernière colonne donne un intervalle de confiance à $95 \%$ pour ces mêmes valeurs. Notons que le calcul des écarts-types est approximatif: il est basé sur une approximation linéaire aux paramètres estimés des relations $(19)^{21}$. Comme l'indique les intervalles de confiance, les estimations ne sont guère très précises, en particulier en ce qui concerne les rendements de densité. Cette imprécision découle bien entendu des difficultés d'estimation des termes quadratiques et d'interactions entre $Y, N$ et $K$ évoquées ci-dessus. Le fait qu'elle soit plus particulièrement marquée pour les rendements de densité est une conséquence de la forte corrélation entre $Y$ et $N$ - Cfr. Section 3.2 - observée dans l'échantillon: si les niveaux de $Y$ et $N$ varient fortement, leur ratio $Y / N$ n'est guère très différent d'une observation à l'autre. Cette collinéarité pose peu de problèmes pour l'évaluation des rendements d'échelle, pour peu que l'on ne s'aventure pas à essayer de les prédire pour des valeurs de $Y$ et $N$ s'écartant du ratio $Y / N$ typique (moyen) observé dans l'échantillon. Elle incite par contre à considérer avec circonspection les rendements de densité reportés.

Bien que peu précis, les rendements d'échelle et de densité présentés dans le Tableau 5 permettent de tirer quelques grands enseignements.

On le sait, des rendements d'échelle croissants (resp. décroissant) impliquent une décroissance (resp. croissance) du coût moyen de distribution (coût par $\mathrm{kWh}$ délivré) lors d'une augmentation de la taille (énergie délivrée et nombre de consommateurs) des firmes. Ainsi, l'accroissement de la taille de la firme de $1 \%$ se traduirait, selon notre estimation, par une augmentation de son coût d'exploitation $^{22}$ de $0.9243 \%$ pour Wavre, $1.0276 \%$ au point moyen et $1.0456 \%$ pour I.E.H. Clairement, des économies d'échelle semblent possibles pour les organismes de petite taille tandis que les organismes de grande taille semblent plutôt être victimes

\footnotetext{
${ }^{21}$ Pour plus de détails, voir Harvey (1990) p.168.

${ }^{22}$ C'est-à-dire (1/RE).
} 
de déséconomies d'échelle.

De façon à nous faire une idée plus précise de la taille optimale (c'est-à-dire la taille pour laquelle $\mathrm{RE}=1$ ) que devrait adopter les organismes de distribution et conformément à la remarque faite ci-dessus, nous avons recalculé les rendements d'échelle pour des valeurs de $Y, N$ et $K$ proportionnelles à leur moyenne d'échantillon (prix des inputs fixés à leur moyenne d'échantillon). La figure 1 représente l'évolution de ces rendements d'échelle en fonction de $N^{23}$.

Figure 1: Rendements d'échelle et taille des organismes distributeurs

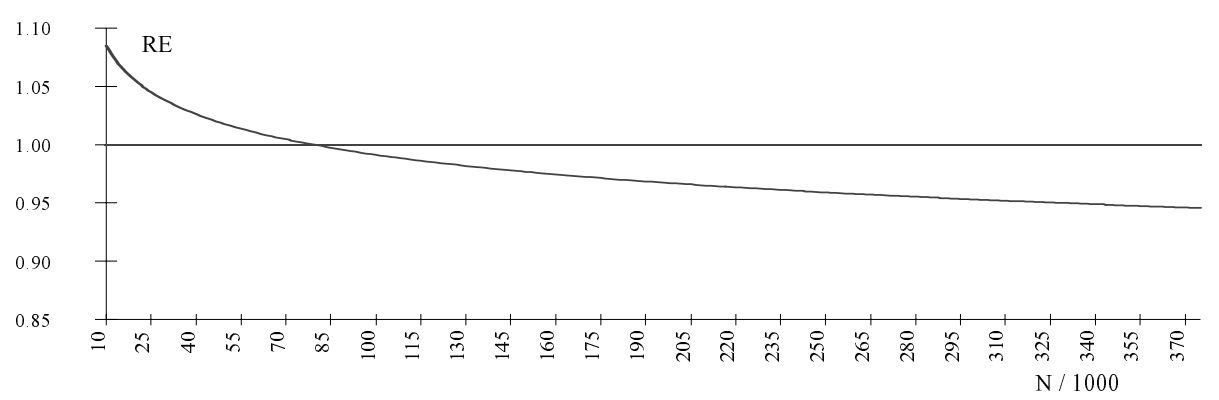

Comme on peut le voir, les économies d'échelle semblent s'épuiser assez rapidement. Selon nos estimations, la taille optimale correspond à des valeurs de $Y, N$ et $K$ égales à plus ou moins $47 \%$ de leur moyenne d'échantillon, soit à un réseau approvisionnant approximativement 80000 abonnés, ce qui est substantiellement supérieur au résultat obtenu par Salvanes et alii (1990) : environ 20000 . Au-delà de cette taille, on assiste à une baisse lente mais régulière des rendements.

80000 raccordements, c'est plus que la plupart des intercommunales pures et que toutes les régies, mais c'est moins que l'écrasante majorité des intercommunales mixtes. En bref, le secteur public semble sous-exploiter les possibilités d'économies d'échelle tandis que le secteur mixte semble au contraire les sur-exploiter. Quoi qu'il en soit, ce résultat fournit une explication économique rationnelle au spectaculaire mouvement de concentration horizontale du secteur observé depuis la seconde guerre mondiale (Cfr. supra). En tout état de cause, il milite en défaveur de l'exploitation de réseaux de distribution au niveau communal, du moins si celle-ci est de taille modeste.

De même que pour les rendements d'échelle, des rendements de densité croissants (resp. décroissant) impliquent une décroissance (resp. croissance) du coût moyen de distribution lors d'une augmentation de l'output physique (énergie délivrée) des firmes, la taille du réseau (mesurée ici par le nombre de clients desservis) restant inchangée. Ainsi, un accroissement de $1 \%$ de la consommation des abonnés se traduirait, selon notre estimation, par une augmentation du coût d'exploitation ${ }^{24}$ de $0.7983 \%$ pour Wavre, $0.9256 \%$ au point moyen et $0.9554 \%$ pour I.E.H.

Ce résultat, classique pour une industrie basée sur l'exploitation d'un réseau mais qui doit être considéré avec réserve étant donné sa forte imprécision statistique, nous semble aisé à comprendre intuitivement. Il indique simplement qu'il est plus facile,

\footnotetext{
${ }^{23} Y$ et $K$ varient proportionnellement selon leur rapport à $N$ au point moyen de l'échantillon, $P_{I}$ et $P_{L}$ sont fixés à leur moyenne d'échantillon.

${ }^{24}$ C'est-à-dire $(1 / \mathrm{RD})$.
} 
et donc moins onéreux, pour un distributeur d'accroître les fournitures d'énergie aux clients existants que de desservir de nouveaux consommateurs. Finalement, si on veut bien admettre, comme nos résultats semblent le suggérer, que ces économies de densité ne sont pas facilement épuisées, il contribue sans doute à expliquer la relative stabilité du prix de vente de l'énergie distribuée, et ce en dépit de la croissance séculaire de la consommation.

Pour conclure, quelques mots concernant l'interprétation à court ou à long terme qu'il convient de donner aux rendements d'échelle et de densité reportés ci-dessus. On sait que les élasticités à court terme (9) et (10) correspondent aux élasticités à long terme (7) si on admet soit que le processus de production sous-jacent est homothétique, soit que la quantité du facteur quasi-fixe $K$ est optimalement ajustée à court terme (Cfr. Section 2.2). Le test de Wald des restrictions (20) rejette l'hypothèse nulle d'homothéticité à un seuil à inférieur à $0.01 \%\left(\chi^{2}(2)=44.39\right.$, Prob. $=0.0000)$. L'hypothèse d'ajustement optimal du facteur quasi-fixe $K$ à court terme peut être testée en suivant l'approche de Nelson (1985). Malheureusement, une telle approche requiert que l'on dispose de données fiables sur le prix du capital. Nous n'en disposons pas. Il convient cependant de noter que cette hypothèse (ajustement optimal de $K$ à court terme) est loin d'être irréaliste. En effet, les divers réseaux étudiés ci-dessous sont en service depuis de longues périodes, et peuvent donc être légitimement supposés bien dimensionnés. Finalement, l'interprétation - court terme ou long terme — des élasticités reportées dépendra de la crédibilité que le lecteur accorde à cette conjecture.

\section{Conclusion}

L'objet de ce papier était d'évaluer de l'opportunité économique (en termes d'efficience-coût) de la politique de délégation de la distribution d'électricité des communes à des organismes externes.

Pour ce faire, les possibilités d'économies d'échelle et de densité au sein du secteur ainsi que les coûts d'exploitation - coûts d'investissements exclus, donc - relatifs des diverses formes institutionnelles existantes ont été évalués sur base de l'estimation par maximum de vraisemblance d'une fonction de coût hédonique à court terme de type translog.

Ce modèle nous a permis de dégager quatre grands résultats, qui tous doivent être appréciés avec les nuances émises ci-dessus lors de leur présentation, en particulier le fait qu'ils découlent de l'étude d'un très petit nombre d'organismes:

1- L'existence de possibilités d'économies d'échelle significatives pour les organismes de petite taille mais de déséconomies d'échelle à partir d'approximativement 80000 abonnés.

2- L'existence de possibilités d'économies de densité appréciables, ce qui laisse présager un impact limité de la croissance séculaire de la consommation sur les coûts de distribution.

3- Une différence systématique de coût d'exploitation de l'ordre de $2.5 \%$ entre les intercommunales mixtes et les sociétés publiques pures (intercommunale et régie).

4- L'absence apparente de possibilités d'économies de gamme, en tout cas dans les 
coûts d'exploitation, au sein des sociétés poursuivant simultanément plusieurs activités de distribution.

Vus de manière qualitative, ces différents résultats suggèrent la conclusion suivante. En termes d'efficience-coût et pour des raisons essentiellement liées à l'existence plus que probable d'économies d'échelle, la politique de délégation des communes, en particulier dans le cas des petites communes, semble économiquement fondée et donc recommandable. Il ne paraît cependant guère opportun de pousser trop loin la concentration horizontale du secteur dans la mesure où les possibilités d'économies d'échelle semble s'épuiser relativement rapidement, et même faire place à des déséconomies d'échelle. En matière de structure institutionnelle, bien que les intercommunales mixtes semblent être légèrement plus efficientes que leurs homologues purement publiques, on se gardera, en l'absence d'informations quant à la qualité du service rendu, de toute conclusion hâtive.

\section{Bibliographie}

Barten E.R. (1969), "Maximum likelihood estimation of a complete system of demand equations", European Economic Review, 1, pp. 7-73.

Caves D.W., Christensen L.R. et Swanson J.A. (1981), "Productivity growth, scale economies and capacity utilization in U.S. railroads 1955-1974", American Economic Review, 71, pp. 994-1002.

Caves D.W., Christensen L.R. et Thretheway M.W. (1984), "Economies of density versus economies of scale: why trunk and local service airline costs differ", Rand Journal of Economics, 15, pp. 471-489.

Davidson R. and MacKinnon J.G. (1993), Estimation and Inference in Econometrics, Oxford University Press, Oxford.

Diewert W.E. et Wales T.J. (1987), "Flexible fonctional forms and Global curvatures conditions", Econometrica, 55 (1), pp. 43-68.

Evrard O. (1993), "Le secteur de la distribution de l'eau en Belgique", Document de travail, 93/11, CIRIEC, Université de Liège.

Evrard O. (1994), "Le secteur de la distribution d'électricité en Belgique", Document de travail, 94/08, CIRIEC, Université de Liège.

FPE (1989), "La distribution d'énergie électrique, situation annuelle", Statistique FPE, Bruxelles.

Fomby T.B., Carter H.R. et Johnson S.R. (1984), Advanced Econometric Methods, Springer-Verlag, New-york.

Harvey A.C. (1990), The Econometric Analysis of Time Series, Philip Allan, Londres.

Heuttner D.A. et Landom J.A. (1978), "Electric utilities: scale economies and diseconomies", Southern Economic Journal, 45, pp. 883-912.

Hjalmarsson L. et Veiderpass A. (1992), "Productivity in swedish electricity retail distribution", Scandinavian Journal of Economics, 94, pp. S193-S205.

Magnus J.R. (1982), "Multivariate error components analysis of linear and nonlinear regression models by maximum likelihood", Journal of Econometrics, 19, 
pp. 239-285.

Nelson R.A. (1985), "Returns to scale from variable and total cost functions, evidence from the electric power industry", Economic Letters, 18, pp. 271-276.

Neuberg L.G. (1977), "Two issues in the municipal ownership of electric power distribution systems", Bell Journal of Economics, 8, pp. 302-323.

Panzar J.C. (1989), "Technological determinants of firm and industry structure", dans Schamlensee R. et Willig R.D., Eds., Handbook of Industrial Organisation, Vol. 1, North Holland, Amsterdam.

Salvanes K.J. et Tjtta S. (1990), "Cost differences in electricity distribution : a multioutput cost function approach with robustness tests of the results", Document de travail, University of Bergen, Norway.

Sheppard R. (1970), Theory of cost and production functions, Princeton University Press, Princeton N.J.

Spady R. et Friedlander A. (1978), "Hedonic cost functions for the regulated trucking industry", Bell Journal of Economics, 9, pp. 159-179.

Theil H. (1971), Principles of econometrics, John Wiley and Sons, Amsterdam.

Weiss L.W. (1975), "Antitrust in the electric power industry", dans Phillips A., Eds., Promoting Competition in Regulated Market, The Brookings Institute, Washingthon D.C.

Wyatt N.S., Brown M.R. and Caragata P.J. (1989), "Performance measures and economies of scale in the New Zealand electricity distribution system", Document de travail, Ministry of Energy, Wellington, New Zealand.

Zellner A., Kmenta L. and Drèze J. (1966), "Specification and estimation of cobbdouglas production function models", Econometrica, 34 (4), pp. 784-795.

Zellner A. (1962), "An efficient method of estimating seemingly unrelated regressions and tests for aggregation bias", Journal of the American Statistical Association, 57 , pp. 348-368. 